Research Paper

\title{
Characteristics of in peripheral blood of 70 hospitalized patients and 8 diarrhea patients with COVID-19
}

\author{
Xiao-Yu Wei"\#, Ding Jing1\#, Bei Jia², Qing Li², Xia-Qia Zhou1, Ming-Feng Gong1, Jing-Bo Zou, Qiang \\ Zhang $^{2}$, Wen-Xiang Huang ${ }^{3 凶}$, Wen-Guang Tian ${ }^{1 凶}$ \\ 1. Department of Infectious Diseases, Yongchuan Hospital of Chongqing Medical University, Chongqing 400016, P.R. China \\ 2. Cancer center, Daping Hospital, The Third Military Medical University, Yuzhong District, Chongqing 400042, P.R. China. \\ 3. Department of Infectious Diseases, The First Affiliated Hospital of Chongqing Medical University, Chongqing 400016, P.R. China. \\ 4. Chongqing Yongchuan District Center for Disease Control and Prevention, Chongqing 400016, P.R. China. \\ \#These authors contributed equally to this work. \\ $\square$ Corresponding authors: Wen-Guang Tian, E-mail: twg9366@163.com.; Wen-Xiang Huang, E-mail:wenxiang_huang@hotmail.com. \\ (c) The author(s). This is an open access article distributed under the terms of the Creative Commons Attribution License (https://creativecommons.org/licenses/by/4.0/). \\ See http://ivyspring.com/terms for full terms and conditions.
}

Received: 2020.03.07; Accepted: 2020.04.28; Published: 2020.05.17

\begin{abstract}
Objective: To analyze the blood test indicators of patients after infection of COVID-19 in Chongqing and analyze the clinical indicators of 8 patients with diarrhea.

Materials and Methods: From January 26, 2019 to February 13, 2020, 70 patients diagnosed with 2019-nCoV according to the World Health Organization interim guidance for NCP and divided into diarrhea and non-diarrhea groups. The laboratory tests liver and kidney function, blood routine, coagulation function, and immune status.

Results: The study population included 70 hospitalized patients with confirmed CONV-2019. NCP patients (43males and 27 females) with a mean age of $48.57 \pm 17.80$ (9 82) years and only $4.3 \%$ of patients have lung-related diseases. The positive rate of ESR, CRP, PT, IL6, lymphocyte count, GGT, Prealbumin and CD4 was more than 50\%. We further analyzed the differences between 8 diarrhea patients and 62 non-diarrhea patients. Among these indicators, only Lymphocyte, CRP, Prealbumin and Cystatin C positive rate is more than $50 \%$. Although there is no statistical difference in GGT, $100 \%$ of the 7 patients tested decreased.

Conclusion: Our data recommended that the ESR, CRP, PT, IL6, lymphocyte count, GGT, prealbumin and CD4 have important value in the diagnosis of COVID-19, and the decrease of GGT may be an important indicator for judging the intestinal dysfunction of patients.
\end{abstract}

Key words: Peripheral blood, COVID-19, GGT, Diarrhea

\section{Introduction}

The new coronavirus (CoV) named by the World Health Organization (WHO) as "COVID-19" led to a pneumonia outbreak that recently started in Wuhan, Hubei Province China [1]. Its outbreak was linked to a large seafood and animal market and investigations are underway to determine the source of infection. Thousands of human infections and many export cases worldwide have been confirmed in China to date [2]. The main source of infection is pneumonia in patients infected with new coronavirus. Respiratory droplets are the main route of transmission and can also be transmitted through contact. People are often vulnerable. The main symptoms are respiratory symptoms such as fever and cough. However, recently, Professor An Ping et al. observed that the first symptom of some patients with pneumonia infected by the new coronavirus is only diarrhea, and it is suspected that the digestive system may also transmit the new coronavirus. At present, the mechanism by which digestive symptoms appear is unclear. The purpose of this study was to analyze the basic conditions of 70 patients with new-type coronavirus in Yongchuan Hospital, to analyze the blood test indicators of patients after infection, and 
the differences in the detection indexes of the infected people in Wuhan and Chongqing, and to analyze the clinical indicators of 8 patients with diarrhea. This will assist in the ability to find specific clinical testing indicators for patients with diarrhea.

\section{Materials and Methods}

\section{Patients}

From January 26, 2019 to February 13, 2020, 70 patients diagnosed with 2019- $\mathrm{nCoV}$ according to the World Health Organization interim guidance for NCP and divided into diarrhea and non-diarrhea groups. All patients were confirmed to be positive for new coronavirus nucleic acid by real-time fluorescent RT-PCR. Infection samples were collected from Chongqing Hospital.

\section{Blood sampling}

Blood samples of the patients were collected by the nurse according to the doctor's order, and all patients were not treated before the blood sampling or did not receive the standardized treatment according to the diagnosis and treatment scheme of NCP.

\section{Blood test}

Liver and kidney function, immunoglobulin complement, and fructosamine machines are all Hitachi 7600 automatic biochemical analyzers from Japan. The blood routine is a Japanese Sysmex $\mathrm{XN}$ 1000 automatic blood analyzer. The coagulation test is a Japanese Sysmex CA7000 automatic coagulation analyzer. Glycated hemoglobin is a Mindray H50 glycated hemoglobin analyzer. Erythrocyte sedimentation is an instrument that I haven't noticed, and I haven't changed it yet.

\section{Statistical analysis}

All statistical analyses were performed using SPSS 20.0 (SPSS Inc., Chicago, IL, USA). Descriptive analyses were performed for categorical variables such as gender. Continuous variables such as inspection results were expressed as $x \pm s$ and compared using the independent samples t-test. $\mathrm{P}<0.05$ was considered as statistically significant.

\section{Results}

\section{Clinical characteristics of all patients}

All patents' clinical specimens for CONV-2019 diagnostic testing were obtained in accordance with the guidelines of the CDC $[3,4]$. A description of the assay and sequence information for the RT-PCR primers and probes are available on the $\mathrm{CDC}$ Laboratory Information website for CONV-2019.

The study population included 70 hospitalized patients with confirmed CONV-2019. NCP patients (43males and 27 females) with a mean age of $48.57 \pm 17.80$ (9 82) years and only $4.3 \%$ of patients have lung-related diseases. Lesion site locations were prominent in both lungs (Table 1).

Table 1. Case characteristics group by 2019 ncov

\begin{tabular}{lll}
\hline Variable & Patient Number & Patient $\%$ \\
\hline Age (mean \pm SD) & $48.57 \pm 17.80$ & \\
Age $(y)$ & 52 & 74.3 \\
$<60$ & 18 & 25.7 \\
$\geq 60$ & & \\
Gender & 43 & 61.4 \\
Male & 27 & 38.6 \\
Female & & \\
Lung related basic diseases & 67 & 95.7 \\
no & 3 & 4.3 \\
yes & & \\
Person category & 29 & 41.4 \\
A & 41 & 58.6 \\
B & & \\
Location of lesion & 10 & 14.3 \\
Right & 7 & 10.0 \\
Left & 46 & 65.7 \\
Both & 7 & 10.0 \\
Normal & & \\
\hline
\end{tabular}

A: Wuhan infected people; B: Infecting people in Chongqing

\section{Blood test indicators of all patients}

All patients were tested for blood routine, liver and kidney function, coagulation, and immune status when they were admitted to the hospital. ESR(39/70), CRP (49/70), PCT (31/58), PT (41/67), IL6 (39/68), Cystatin C (23/68) were significantly elevated and the lymphocyte count (39/70), Prealbumin (39/67), GGT (54/70), CD4 (50/66), CD8 (24/70), T cell $(26 / 65)$ were significantly decreased and the abnormal rate was more than $30 \%$. At the same time, the positive rate of ESR, CRP, PT, IL6, lymphocyte count, GGT, Prealbumin and CD4 is more than $50 \%$. These indicators may have more important clinical significance in the diagnosis process (Table 2).

Table 2. Differences in clinical blood test indicators among all patients

\begin{tabular}{lllll}
\hline Variable & $\begin{array}{l}\text { Normal NO. } \\
(\text { mean } \pm \text { SD })\end{array}$ & $\begin{array}{l}\text { High NO. } \\
(\text { mean } \pm \text { SD })\end{array}$ & $\begin{array}{l}\text { Low NO. } \\
(\text { mean } \pm \text { SD })\end{array}$ & $\begin{array}{l}\text { P value } \\
\text { (mean) }\end{array}$ \\
\hline WBC & $59(5.35 \pm 1.53)$ & & $11(2.90 \pm 0.53)$ & b, $P=0.000$ \\
Lymphocyte & $31(1.52 \pm 0.36)$ & & $39(0.76 \pm 0.23)$ & b, $P=0.000$ \\
Neutrophil cell & $63(3.5 \pm 1.09)$ & $3(7.55 \pm 3.61)$ & $4(1.23 \pm 0.28)$ & $\begin{array}{l}\text { a, } P=0.000 \\
\text { b, } P=0.000\end{array}$ \\
N\% (40-75) & $57(64.81 \pm 8.56)$ & $13(81.68 \pm 6.20)$ & & a, $P=0.000$ \\
PLT & $55(195.56 \pm 51.03)$ & $3(325 \pm 8.49)$ & $12(95.75 \pm 19.07)$ & a, $P=0.000$ \\
& & & & b $P=0.000$ \\
HB & $54(105.37 \pm 9.10)$ & $8(157.27 \pm 6.25)$ & $8(133.39 \pm 9.39)$ & a, $P=0.000$ \\
& & & & b, $P=0.000$ \\
CRP & $31(3.11 \pm 2.82)$ & $39(36.26 \pm 27.79)$ & & a, $P=0.000$ \\
ESR & $21(10.26 \pm 4.25)$ & $49(60.80 \pm 29.36)$ & & a, $P=0.000$ \\
PCT & $27(0.06 \pm 0.03)$ & $31(0.12 \pm 0.17)$ & & a, $P=0.041$ \\
IL6 & $29(2.66 \pm 1.58)$ & $39(23.91 \pm 19.80)$ & a, $P=0.000$ \\
BNT & $63(76.13 \pm 83.57)$ & $5(500 \pm 320.20)$ & & a, $P=0.041$ \\
PT & $26(12.07 \pm 0.66)$ & $41(14.69 \pm 1.39)$ & & a, $P=0.000$ \\
PTA & $62(88.10 \pm 8.69)$ & $6(61.83 \pm 2.40)$ & & a, $P=0.000$ \\
\hline
\end{tabular}




\begin{tabular}{|c|c|c|c|c|}
\hline d-dimer & $59(0.35 \pm 0.23)$ & $3(1.30 \pm 0.17)$ & & a, $P=0.000$ \\
\hline ALT & $62(24.03 \pm 9.23)$ & $8(89.75 \pm 37.72)$ & & a, $P=0.002$ \\
\hline AST & $55(25.07 \pm 5.90)$ & $15(56.73 \pm 29.20)$ & & a, $P=0.001$ \\
\hline GGT & $9(83.67 \pm 27.69)$ & $7(194.86 \pm 51.04)$ & $54(26.72 \pm 18.57)$ & $\begin{array}{l}\text { a, } P=0.001 \\
\text { b, } P=0.000\end{array}$ \\
\hline Prealbumin & $28(242 \pm 28.17)$ & & $\begin{array}{l}39 \\
(131.51 \pm 41.87)\end{array}$ & b, $P=0.000$ \\
\hline ALB & $58(42.33 \pm 4.11)$ & & $11(33.38 \pm 1.42)$ & b, $P=0.000$ \\
\hline CK & $51(66.67 \pm 30.20)$ & $11(14.64 \pm 49.22)$ & & b, $P=0.008$ \\
\hline CK-MB & $53(0.46 \pm 0.45)$ & $9(5.97 \pm 2.05)$ & & a, $P=0.043$ \\
\hline LDH & $57(470.37 \pm 96.41)$ & $\begin{array}{l}7 \\
(749.59 \pm 124.89)\end{array}$ & & a, $P=0.001$ \\
\hline Myoglobin & $60(31.05 \pm 14.61)$ & $\begin{array}{l}8 \\
(313.45 \pm 584.95)\end{array}$ & & a, $P=0.214$ \\
\hline Creatinine & $60(67.62 \pm 13.23)$ & $2(128 \pm 8.49)$ & $8(38.75 \pm 3.85)$ & $\begin{array}{l}\text { a, } P=0.000 \\
\text { b, } P=0.000\end{array}$ \\
\hline Urea nitrogen & $60(4.53 \pm 1.17)$ & & $9(2.52 \pm 0.29)$ & b, $P=0.000$ \\
\hline $\begin{array}{l}\text { a1 } \\
\text { microglobulin }\end{array}$ & $57(21.84 \pm 4.57)$ & $11(34.69 \pm 4.44)$ & & a, $P=0.000$ \\
\hline Cystatin C & $45(0.95 \pm 0.11)$ & $23(1.34 \pm 0.32)$ & & a, $P=0.000$ \\
\hline $\begin{array}{l}\text { Glycated } \\
\text { hemoglobin }\end{array}$ & $40(5.74 \pm 0.63)$ & $10(8.14 \pm 2.80)$ & & a, $P=0.027$ \\
\hline IgG & $52(11.62 \pm 2.06)$ & $10(18.49 \pm 3.12)$ & $2(7.2 \pm 0.85)$ & $\begin{array}{l}\text { a, } P=0.000 \\
\text { b, } P=0.004\end{array}$ \\
\hline $\operatorname{Ig} \mathrm{A}$ & $55(1.95 \pm 0.53)$ & $9(4.53 \pm 1.27)$ & & a, $P=0.000$ \\
\hline IgM & $61(1.22 \pm 0.43)$ & $2(2.44 \pm 0.08)$ & $1(-)$ & a, $P=0.000$ \\
\hline $\mathrm{C} 3$ & $62(1.32 \pm 0.17)$ & $2(1.75 \pm 0.05)$ & & a, $P=0.001$ \\
\hline $\mathrm{C} 4$ & $47(0.31 \pm 0.06)$ & $15(0.48 \pm 0.10)$ & $2(0.16 \pm 0.01)$ & $\begin{array}{l}\text { a, } P=0.000 \\
\text { b, } P=0.001\end{array}$ \\
\hline $\mathrm{CD} 4$ & $16(715.69 \pm 161.46)$ & & $\begin{array}{l}50 \\
(314.78 \pm 126.11)\end{array}$ & b, $P=0.000$ \\
\hline CD8 & $46(365.47 \pm 175.03)$ & & $\begin{array}{l}24 \\
(126.21 \pm 39.04)\end{array}$ & b, $P=0.000$ \\
\hline $\mathrm{T}$ cell & $39(917.44 \pm 333.36)$ & & $\begin{array}{l}26 \\
(403.31 \pm 180.52)\end{array}$ & b, $P=0.000$ \\
\hline
\end{tabular}

Note: a: high vs normal, b: low vs normal.

\section{Comparison of diarrhea and non-diarrhea populations}

However, the clinical examination of patients with diarrhea as the main symptom is not clear. We further analyzed the differences between 8 diarrhea patients and 62 non-diarrhea patients. The Eight patients had diarrhea as the first symptom, and the throat swab test was negative but the anal swab test was positive, and except for one patient who had slight inflammation of the lungs when admitted, the other patients had no obvious inflammatory changes in the lungs (Figure 1). There was no significant difference between the two groups in the general situation of age, gender, medical history, etc. (Table 3). Comparison of clinical test indicators between the two groups of patients found that GGT (diarrhea vs. non-diarrhea: $20.75 \pm 14.12$ vs. $54.74 \pm 59.61 ; \mathrm{P}=0.000$ ) was significantly different. The results indicate that GGT may be a deliberate detection index for patients with diarrhea. Further analysis of clinical test indicators of 8 patients showed that the Lymphocyte (4/8), PLT(2/8), CRP (4/8), PT (2/8), Prealbumin (5/8), Creatinine (2/8), Cystatin C (4/8), C4 (3/8) and CD8 (3/8) had significant differences $(\mathrm{P}<0.05)$. Among these indicators, only Lymphocyte, CRP, Prealbumin and Cystatin $C$ positive rate is more than $50 \%$. Although there is no statistical difference in GGT, $100 \%$ of the 7 patients tested decreased, so it further illustrates the important detection value of GGT in patients with diarrhea.

Table 3. Comparison of clinical data between diarrhea and non-diarrhea

\begin{tabular}{llll}
\hline Variable & Diarrhea $(\mathrm{n}=8)$ & Non-diarrhea $(\mathrm{n}=62)$ & P value \\
\hline $\begin{array}{l}\text { Age }(\text { mean } \pm \text { SD) } \\
\text { Age }(\mathrm{y})\end{array}$ & $42.38 \pm 17.74$ & $49.37 \pm 17.80$ & 0.299 \\
$<60$ & 8 & & \\
$\geq 60$ & 0 & 43 & 0.097 \\
Gender & & 19 & \\
Male & 5 & & 0.714 \\
Female & 3 & 39 & \\
Lung related basic diseases & & 23 & \\
no & 8 & & \\
yes & 0 & 59 & \\
Location of lesion & & 3 & 0.471 \\
Right & 0 & & \\
Left & 0 & 12 & \\
Both & 1 & 4 & \\
Normal & 7 & 38 & \\
\hline
\end{tabular}

\section{Date}

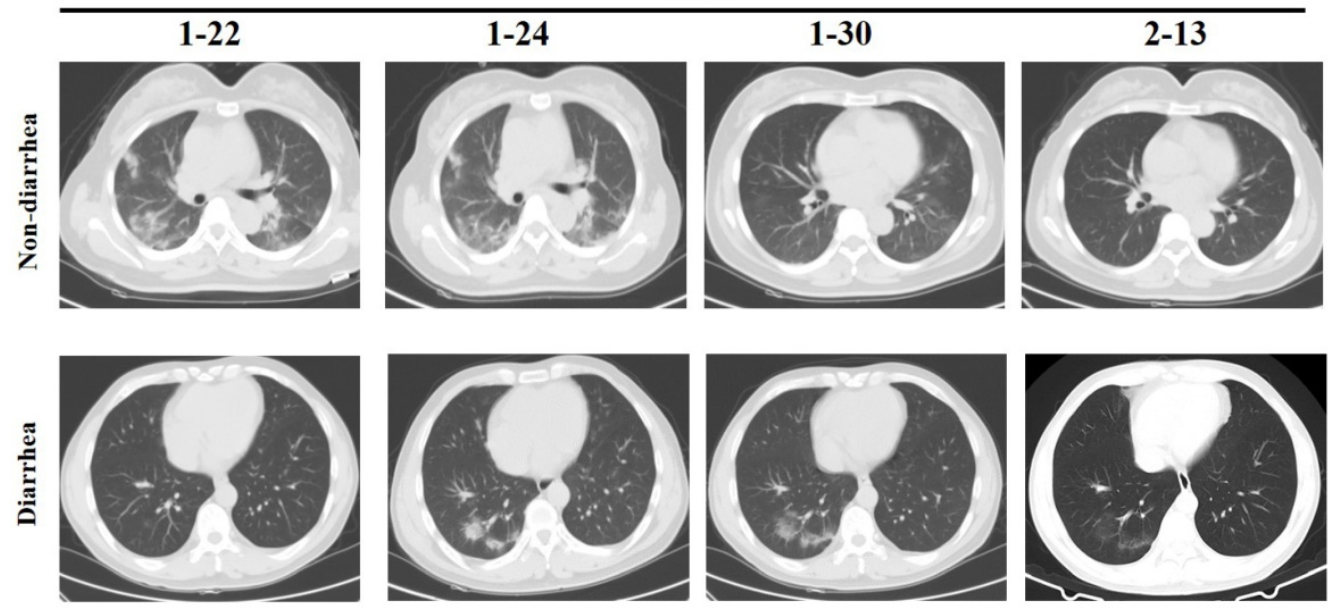

Figure 1. CT of chest in patients with diarrhea and non-diarrhea. 
Table 4. Comparison of clinical indicators of diarrhea and non-diarrhea

\begin{tabular}{|c|c|c|c|}
\hline Variable & Diarrhea & No diarrhea & $P$ value \\
\hline WBC & $4.50 \pm 1.18$ & $5.21 \pm 2.08$ & 0.353 \\
\hline Lymphocyte & $1.17 \pm 0.49$ & $1.08 \pm 0.50$ & 0.635 \\
\hline Neutrophil cell & $2.96 \pm 1.35$ & $3.61 \pm 1.80$ & 0.327 \\
\hline N\% & $63.25 \pm 15.19$ & $67.84 \pm 10.30$ & 0.266 \\
\hline PLT & $182.63 \pm 46.88$ & $180.81 \pm 66.96$ & 0.941 \\
\hline HB & $132.50 \pm 13.94$ & $134.13 \pm 16.46$ & 0.790 \\
\hline CRP & $22.36 \pm 23.17$ & $21.48 \pm 27.10$ & 0.931 \\
\hline ESR & $41.50 \pm 47.48$ & $47.60 \pm 32.05$ & 0.635 \\
\hline PCT & $0.22 \pm 0.36$ & $0.07 \pm 0.04$ & 0.276 \\
\hline IL6 & $11.55 \pm 21.95$ & $15.06 \pm 17.94$ & 0.614 \\
\hline BNT & $73.63 \pm 83.54$ & $98.9 \pm 141.59$ & 0.624 \\
\hline PT & $12.93 \pm 1.60$ & $13.74 \pm 1.84$ & 0.241 \\
\hline PTA & $87.65 \pm 7.21$ & $83.67 \pm 16.34$ & 0.502 \\
\hline d-dimer & $0.32 \pm 0.24$ & $0.41 \pm 0.31$ & 0.456 \\
\hline ALT & $21.13 \pm 17.46$ & $32.89 \pm 26.43$ & 0.227 \\
\hline AST & $27.00 \pm 16.54$ & $32.48 \pm 19.63$ & 0.453 \\
\hline GGT & $20.75 \pm 14.12$ & $54.74 \pm 59.61$ & 0.000 \\
\hline Prealbumin & $169.63 \pm 73.49$ & $178.7 \pm 65.47$ & 0.716 \\
\hline ALB & $42.21 \pm 5.84$ & $40.73 \pm 4.95$ & 0.438 \\
\hline CK & $67.63 \pm 40.13$ & $117.04 \pm 150.14$ & 0.361 \\
\hline CK-MB & $0.34 \pm 0.20$ & $0.81 \pm 1.44$ & 0.364 \\
\hline LDH & $521.38 \pm 189.60$ & $532.76 \pm 169.68$ & 0.861 \\
\hline Myoglobin & $27.49 \pm 8.54$ & $69.18 \pm 223.90$ & 0.603 \\
\hline Creatinine & $62.86 \pm 17.04$ & $66.45 \pm 19.06$ & 0.615 \\
\hline Urea nitrogen & $3.98 \pm 1.05$ & $4.39 \pm 1.46$ & 0.446 \\
\hline a1 microglobulin & $23.13 \pm 6.27$ & $24.03 \pm 6.65$ & 0.718 \\
\hline Cystatin C & $1.02 \pm 0.15$ & $1.09 \pm 0.28$ & 0.510 \\
\hline Glycated hemoglobin & $5.81 \pm 0.30$ & $6.15 \pm 1.98$ & 0.676 \\
\hline $\operatorname{IgG}$ & $11.77 \pm 1.95$ & $12.65 \pm 3.61$ & 0.530 \\
\hline $\operatorname{IgA}$ & $2.62 \pm 1.36$ & $2.30 \pm 1.09$ & 0.484 \\
\hline IgM & $1.57 \pm 0.55$ & $1.21 \pm 0.46$ & 0.061 \\
\hline $\mathrm{C} 3$ & $1.29 \pm 0.12$ & $1.34 \pm 0.19$ & 0.506 \\
\hline $\mathrm{C} 4$ & $0.37 \pm 0.05$ & $0.34 \pm 0.11$ & 0.557 \\
\hline CD4 & $404.63 \pm 153.05$ & $431.19 \pm 265.49$ & 0.784 \\
\hline CD8 & $319.50 \pm 210.50$ & $274.37 \pm 179.99$ & 0.516 \\
\hline $\mathrm{T}$ cell & $764.13 \pm 349.30$ & $733.25 \pm 438.44$ & 0.849 \\
\hline
\end{tabular}

\section{Discussion}

So far, there is no vaccine or specific antiviral treatment for this emerging infectious disease, and effective control still depends mainly on early diagnosis, patient isolation and close contact monitoring [3]. Therefore, reliable and accurate diagnostic methods play a vital role in the field of disease control and prevention. However, some patients with diarrhea have a false-negative nucleic acid test. So, finding indicators that can predict gut symptoms in patients has important clinical significance. Our data found that the positive rate of ESR, CRP, PT, IL6, lymphocyte count, GGT, Prealbumin and CD4 was more than $50 \%$. In addition, it was found that the Lymphocyte, PLT, CRP, PT, Prealbumin, Creatinine, Cystatin C, C4 and CD8 have clinical significance for patients in the diagnosis of diarrhea. GGT reduction is probably the most important indicator. Taken together, consistent with previous research $[2,5]$, the ESR, CRP, PT, IL6, lymphocyte count, GGT, Prealbumin and CD4 have important value in the diagnosis of COVID-19, and the decrease of GGT may be an important indicator for judging the intestinal dysfunction of patients.

Table 5. Analysis of clinical detection indicators in patients with diarrhea

\begin{tabular}{|c|c|c|c|c|}
\hline Variable & $\begin{array}{l}\text { Normal NO. } \\
(\text { mean } \pm S D)\end{array}$ & $\begin{array}{l}\text { High NO. } \\
(\text { mean } \pm S D)\end{array}$ & $\begin{array}{l}\text { Low NO. } \\
(\text { mean } \pm S D)\end{array}$ & $P$ value \\
\hline WBC & $7(4.75 \pm 1.00)$ & & & \\
\hline Lymphocyte & $4(1.51 \pm 0.29)$ & & $4(0.83 \pm 0.39)$ & a, $P=0.032$ \\
\hline Neutrophil cell & $6(3.43 \pm 1.21)$ & & $2(1.53 \pm 0.21)$ & a, $P=0.081$ \\
\hline NEU & $7(59.03 \pm 10.15)$ & $1(92.8)$ & & \\
\hline PLT & $6(204.67 \pm 27.19)$ & & $2(116.50 \pm 4.95)$ & b, $P=0.005$ \\
\hline HB & $4(135.57 \pm 11.77)$ & & & \\
\hline CRP & $4(2.60 \pm 2.73)$ & $4(42.13 \pm 14.29)$ & & a, $P=0.010$ \\
\hline ESR & $5(12.2 \pm 6.22)$ & $3(90.33 \pm 45.72)$ & & a, $P=0.096$ \\
\hline PCT & $4(<0.05)$ & $4(0.40 \pm 0.47)$ & & a, $P=0.235$ \\
\hline IL6 & $6(<1.5)$ & $3(34.44 \pm 25.14)$ & & a, $P=0.151$ \\
\hline BNT & $8(73.63 \pm 83.54)$ & & & \\
\hline PT & $6(12.15 \pm 0.83)$ & $2(15.25 \pm 0.35)$ & & a, $P=0.003$ \\
\hline PTA & $8(87.65 \pm 7.24)$ & & & \\
\hline D-dimer & $8(0.32 \pm 0.24)$ & & & \\
\hline ALT & $7(15 \pm 2.31)$ & & & \\
\hline AST & $7(21.43 \pm 5.44)$ & & & \\
\hline GGT & & & $7(15.86 \pm 3.02)$ & \\
\hline Prealbumin & $3(248.33 \pm 28.10)$ & & $5(122.40 \pm 40.30)$ & a, $P=0.003$ \\
\hline ALB & $7(43.26 \pm 5.44)$ & & & \\
\hline CK & $7(54.57 \pm 16.99)$ & & & \\
\hline CK-MB & $8(0.34 \pm 0.20)$ & & & \\
\hline LDH & $6(423.83 \pm 61.75)$ & $2(814 \pm 65.05)$ & & \\
\hline Myoglobin & $8(27.49 \pm 8.54)$ & & & \\
\hline Creatinine & $6(71.00 \pm 9.23)$ & & $2(38.5 \pm 4.95)$ & b, $P=0.004$ \\
\hline Urea nitrogen & $7(4.27 \pm 0.71)$ & & & \\
\hline $\begin{array}{l}\text { a1 } \\
\text { microglobulin }\end{array}$ & $7(21.53 \pm 4.69)$ & & & \\
\hline Cystatin C & $4(0.9 \pm 0,09)$ & $4(1.15 \pm 0.06)$ & & a, $P=0.005$ \\
\hline $\begin{array}{l}\text { Glycated } \\
\text { hemoglobin }\end{array}$ & $6(5.81 \pm 0.30)$ & & & \\
\hline $\operatorname{IgG}$ & $6(12.2 \pm 1.74)$ & & & \\
\hline $\operatorname{IgA}$ & $5(1.93 \pm 0.48)$ & $2(4.34 \pm 1.14)$ & & a, $P=0.237$ \\
\hline IgM & $6(1.41 \pm 0.41)$ & $1(2.5)$ & & \\
\hline $\mathrm{C} 3$ & $7(1.23 \pm 0.12)$ & & & \\
\hline $\mathrm{C} 4$ & $5(0.34 \pm 0.03)$ & $3(0.43 \pm 0.01)$ & & a, $P=0.017$ \\
\hline CD4 & $4(509.05 \pm 9.00)$ & & $\begin{array}{l}4 \\
(340.80 \pm 165.40)\end{array}$ & b, $P=0.084$ \\
\hline CD8 & $\begin{array}{l}5 \\
(429.20 \pm 189.63)\end{array}$ & & $3(136.67 \pm 54.27)$ & b, $P=0.044$ \\
\hline T cell & $\begin{array}{l}7 \\
(855.14 \pm 255.00)\end{array}$ & & $1(127)$ & \\
\hline
\end{tabular}

Note: a: high vs normal, b: low vs normal.

\section{Clinical Perspectives}

Our study analyzed the detection of 70 patients with COVID-19 infection in Chongqing, and specifically analyzed the blood test of 8 patients with diarrhea as the first symptom. The decrease of GGT may be an important indicator for judging the intestinal dysfunction of patients. It has clinical significance for the diagnosis and treatment of diarrhea patients.

\section{Acknowledgments}

The study was supported by Chongqing Medical University New Coronary Virus Pneumonia Disease Progression and Prognosis Model Establishment Project (20200212-10). 
Table 6. Test indicator description and normal value range

Blood routine

Leukocytes $\left(\times 10^{9}\right.$ per $\mathrm{L}$, normal range $\left.3.5-9.5\right)$

Neutrophils $\left(\times 10^{9}\right.$ per L, normal range $\left.1.8-6.3\right)$

Neutrophil percentage (Neutrophil percentage 40-75)

Lymphocytes $\left(\times 10^{9}\right.$ per L, normal range 1.1-3.2)

Platelets $\left(\times 10^{9}\right.$ per $\mathrm{L}$, normal range $\left.125-350\right)$

Hemoglobin (g/L, normal range Male 130-175; Female 115-150)

Brain natriuretic peptide $(\mathrm{pg} / \mathrm{ml}$, normal range $1-125)$

Coagulation function

Prothrombin time (s, normal range 11-14.5)

D-dimer (ug/L, normal range 0.00-0.5)

Prothrombin time activity (\%, normal range 70-120)

Blood biochemistry

Alanine aminotransferase (U/L, normal range Male 9-50; Female 7-40)

Aspartate aminotransferase (U/L, normal range Male 15-40; Female 13-35)

Gamma glutaminase (GGT) (U/L, normal range Male 10-60; Female 7-45)

Prealbumin (mg/L, normal range 200-400)

Albumin (G/L, normal range 35-55)

Creatine kinase (U/L, normal range Male 55-170; Female 30-135)

Creatine kinase isoenzyme $(\mathrm{ng} / \mathrm{ml}$, normal range 0.0-3.4)
Lactate dehydrogenase (U/L, normal range 313-618)

Myoglobin (ng/mL, normal range Male 0-121; Female 0-61.5)

Serum Creatinine (umol/L, normal range Male 53-123; Female 62-106)

Blood Urea nitrogen (mmol/L, normal range 2.86-8.20)

a1 microglobulin $(\mathrm{mg} / \mathrm{L}$, normal range $10-30)$

Cystatin C (mg/L, normal range 0.51-1.09)

Glycated hemoglobin (\%, normal range 4.5-6.3)

Immunoglobulin complement $\mathrm{G}(\mathrm{G} / \mathrm{L}$, normal range 8-16)

Immunoglobulin complement A (G/L, normal range 0.7-3.3)

Immunoglobulin complement $\mathrm{M}(\mathrm{G} / \mathrm{L}$, normal range 0.5-2.2)

Complement 3 (G/L, normal range 0.8-1.6)

Complement 4 (G/L, normal range 0.2-0.4)

Infection-related factors

Erythrocyte sedimentation rate $(\mathrm{mm} / \mathrm{h}$, normal range Male 0-15; Female 0-20)

C-reactive protein $(\mathrm{mg} / \mathrm{L}$, normal range 0-10)

Procalcitonin $(\mathrm{ng} / \mathrm{ml}$, normal range $0-0.05)$

Interleukin -6 (Pg/ml, normal range $0-7)$

CD4 T-cell (per ul, normal range 493-1191)

CD8 T-cell (per ul, normal range 182-785)

Total T-Cell (per ul, normal range 644-2201)

\section{The data availability statement}

All data gathered and included in the present study are available and can be provided by the corresponding author upon reasonable request.

\section{Ethics approval}

This study was approved by the ethical committee of Yongchuan Hospital of Chongqing Medical University and informed consent was obtained from each patient.

\section{Competing Interests}

The authors have declared that no competing interest exists.

\section{References}

1. Huang C, Wang Y, Li X et al. Clinical features of patients infected with 2019 novel coronavirus in Wuhan, China. Lancet. 2020;. 395(10223):497-506.

2. Chen N, Zhou M, Dong $X$ et al. Epidemiological and clinical characteristics of 99 cases of 2019 novel coronavirus pneumonia in Wuhan, China: a descriptive study. Lancet. 2020;395(10223):507-513.

3. CfDCa, P. Interim Guidelines for Collecting, Handling, and Testing Clinical Specimens from Persons Under Investigation (PUIs) for Coronavirus Disease 2019 (COVID-19). Interim Guidelines for Collecting, Handling, and Testing Clinical Specimens from Persons Under Investigation (PUIs) for Coronavirus Disease 2019 (COVID-19). https://www.cdc.gov/coronavirus/2019-ncov/ lab/guidelines-clinical-specimens.html, 2020.

4. CNH C. the Novel Coronavirus Pneumonia Prevention and Control guideline. http://www.nhc.gov.cn/yzygi/s7653p/202002/3b09b894ac9b4204a79db5b89 12d4440.shtml, 2020.

5. Wang D, Hu B, Hu C, et al. Clinical Characteristics of 138 Hospitalized Patients With 2019 Novel Coronavirus-Infected Pneumonia in Wuhan, China. JAMA, 2020. 